\title{
Cues associated with frustrative nonreward do not necessarily have aversive motivational properties
}

\author{
RODERICK WONG, UNIVERSITY OF BRITISH COLUMBIA \\ WILLIAM B. PAVLIK, RUTGERS UNIVERSITY
}

Rats were given 120 discrimination training trials in a Y-maze with black and white goal boxes. The Ss were divided into three groups: $P$ was tested in a runway from a start box of the color associated with reward, $\mathrm{N}$ was tested from a start box of the color associated with nonreward, and $\mathrm{C}$ was tested from a box painted gray. Starting and running speeds of each $S$ were obtained for each block of four trials. Results indicate that $\mathrm{C}$ ran consistently faster out of the start box than did either $\mathrm{P}$ or $\mathrm{N}$. There were no significant differences between these three groups in running speed.

In an attempt to demonstrate the similarities of punishment and frustrative nonreward in their effects on instrumental reward learning, Wagner (1963) has shown that cues associated with frustrative nonreward, through their elicitation of anticipatory frustration, have aversive motivational properties. This phenomenon was demonstrated by the fact that the presence of cues previously paired with frustrative nonreward potentiated an unconditioned startle response, and that the cessation of such cues served as reinforcement for a simple hurdle-crossing response.

As a means of testing the generality of the results obtained by Wagner (1963) this study is concerned with investigating the consequences of the generalization of the fractional anticipatory goal responses $\left(\mathbf{r}_{\mathrm{g}}\right)$ and the fractional anticipatory frustrative responses $\left(\mathbf{r}_{\mathrm{f}}\right)$ from one experimental situation to another. This was done by training $S s$ to discriminate black from white goal boxes of a Y-maze. Some Ss were consistently rewarded in the black goal box and consistently not rewarded in the white goal box. Other Ss received the opposite treatment.

The Ss then were tested in a runway with a neutral (gray color) alley and goal box. One group was placed in a start box which was painted the same color as the goal box in which the rat was rewarded in the $\mathrm{Y}$-maze situation. During the 10-sec. period in which $S$ was confined in the start box, it is expected that $S$ would show a tendency to remain in the start box, since the color of the box had been associated with reward.

A second group, placed in a start box painted the same color as the goal box in which $S$ previously was not rewarded, should have developed considerable avoidance tendencies as a result of being confined in this box for the 10-sec. interval. A third group which was placed in a neutral gray start box, served as a control group.

Method

Thirty-two female albino rats were used as Ss. They were about 90 days old at the beginning of the experi- ment, at which time they were divided into three groups; 11 Ss were randomly assigned to each of the two experimental groups and the remaining 10 Ss were assigned to the control group.

Two apparatuses were used in the experiment. The first, used for discrimination learning, was a Y-maze, consisting of a 29-in. stem, 12-in. arms, and 11-in. start box and goal boxes. The stem and arms were painted a flat medium gray; one of the detachable goal boxes was painted white and the other black.

The second apparatus, used in the daily test sessions, was located in a different room. It consisted of a 24-in. straight runway connecting a 12-in. start box with a 12-in. goal box, both of which were painted gray. Three different colors of start boxes were used. These were painted white, black and gray. Photoelectric circuits provided measures of starting time, and running time in the stem.

The Ss were run under 22-hr. food deprivation and had access to water at all times. Each $S$ in all three groups received a block of four trials daily on the discrimination task. A block consisted of one free trial followed by three forced trials. In Group P, five Ss received a $0.33-\mathrm{gm}$ pellet of wet mash if they chose the white goal box, and six Ss were reinforced for choosing the black goal box. In Group N, six Ss were rewarded for response to a white stimulus, and five $S$ had black as their positive stimulus. In Group $\mathrm{C}$, five Ss were rewarded in the white goal box, and five Ss were fed if they chose the black goal box. The location of the positive goal box was varied randomly from side to side on both free and forced trials. The blocks were arranged so that each $\mathrm{S}$ ran into the positive goal box for two trials and into the negative box for the other two trials each day. The order of these trials was randomly assigned.

After 60 trials (15 days) of discrimination learning, Ss received reversal discrimination training. The reversal series consisted of one free and three forced trials per day, for a period of another 15 days.

Following each day's discrimination trials, Ss were immediately transported to the second experimental room where each was given four trials in the runway. On each trial $S$ was placed into the start box, and after a 10-sec. interval, the door was opened. Each response was reinforced by $0.33 \mathrm{gm}$ of wet mash.

A total of 120 trials was given in 30 consecutive days. For Group $\mathbf{P}$ the start box of the runway was always the same color as the positive stimulus during the initial discrimination training; for Group $\mathrm{N}$ the start box of the runway was always the same color as the 
negative stimulus during the initial discrimination training, and for Group $\mathrm{C}$ the start box was always a neutral gray.

\section{Results}

An analysis of variance was performed on the trials to criterion among the three groups in their Y-maze performance. The F-value for original discrimination was 2.09, and the reversal discrimination scores yielded an $\mathrm{F}$ of 2.08 . None of these was significant at the .05 level.

For the analysis of the runway data, all time measures were converted to speed scores (10/1at.), and a mean speed score was computed for each $\mathrm{S}$ for each block of four trials. Figure 1 presents the startingspeed acquisition curves for the three groups.

Differences in the mean starting speed scores between pairs of groups during the last four days of discrimination learning (Blocks 12, 13, 14 and 15) and during the last four days of reversal discrimination learning (Blocks 27, 28, 29 and 30) were evaluated by Tukey's method of testing all possible pairs of means. Comparisons between Groups $\mathrm{P}$ and $\mathrm{C}$ during Blocks 12-15 yielded $t$-values indicating that with the exception of Block 13, all of these differences were significant $(p<.05)$. Similar comparisons made between Groups

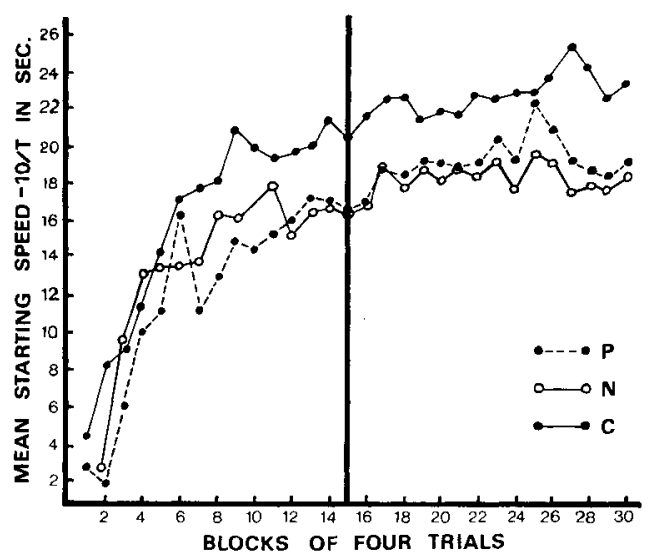

Fig. 1. Mean starting-syeed from runway. The vertical line through Block 15 indicates the beginning of the discrimination reversal trials.
$\mathrm{N}$ and $\mathrm{C}$ during original discrimination yielded Tukey's t-values which approached significance at the .05 level.

During the last four blocks of reversal discrimination learning, comparisons between Groups $\mathrm{P}$ and $\mathrm{C}$, and $\mathrm{N}$ and $\mathrm{C}$ showed differences which were significant $(p<.05)$. The results of t-tests between Groups $P$ and $\mathrm{N}$ produced values less than unity during both the original and the reversal discrimination trials.

Similar analyses were conducted on the runningspeed scores, and the results indicated no differences at the .05 level.

\section{Discussion}

The data do not support the predictions which were based on the notion that conditioned frustration can act as a learned drive. In fact, the starting-speed data are contrary to this prediction; Ss in the control group showed faster starting-speeds than either of the experimental groups.

However, an alternative prediction to the one proposed prior to the study can be made from the same set of theoretical considerations. The occurrence of $\mathrm{r}_{\mathrm{f}}-\mathrm{s}_{\mathrm{f}}$ of $\mathrm{Ss}$ in Group $\mathrm{N}$ during the time when they were confined in the start box could have produced competing response tendencies that interfered with an optimal occurrence of the running response. In this respect, it is important to note that there was no significant difference between Groups $\mathrm{N}$ and $\mathrm{C}$ in running speed which was measured in a portion of the runway which was not associated with frustrative cues. Because $\mathbf{r}_{\mathbf{f}}-s_{f}$ could elicit competing response tendencies that interfere with the instrumental locomotor response, it could be predicted that Ss in Group $N$ would leave the start box more slowly than those in Group $\mathrm{C}$.

A similar explanation could be advanced for the inferior performance of Group $P$ relative to Group C. During discrimination training, Group $P$ has learned to approach a box of a certain color; during runway testing, they must leave a box of the same color. Such a situation is apt to produce conflicting response tendencies, and thus depress the performance of the running response.

\section{Reference}

Wagner, A. R. Conditioned frustration as a learned drive. J. exp. Psychol., 1963, 66, 142-148. 